\title{
Correction to: Effect of paclobutrazol, a potential growth regulator on stalk mechanical strength, lignin accumulation and its relation with lodging resistance of maize
}

Muhammad Kamran ${ }^{1,2} \cdot$ Wenwen Cui $^{1,2} \cdot$ Irshad Ahmad $^{1,2} \cdot$ Xiangping Meng ${ }^{1,2} \cdot$ Xudong Zhang $^{1,2} \cdot$ Wennan Su ${ }^{1,2}$. Junzhi Chen ${ }^{1,2}$. Shakeel Ahmad ${ }^{1,2}$. Shah Fahad ${ }^{3}$. Qingfang Han ${ }^{1,2}$. Tiening Liu ${ }^{1,2}$

Published online: 9 January 2018

(c) Springer Science+Business Media B.V., part of Springer Nature 2018

Correction to: Plant Growth Regul

https://doi.org/10.1007/s10725-017-0342-8

In the original publication of the article, the Table 3 was wrongly published. The correct Table 3 is provided here.

The original article can be found online at https://doi.org/10.1007/ s10725-017-0342-8.

Qingfang Han

hanqf88@nwafu.edu.cn

$\triangle$ Tiening Liu

liutiening0319@163.com

1 College of Agronomy, Key Laboratory of Crop Physio-Ecology and Tillage in Northwestern Loess Plateau,

Minister of Agriculture, Northwest A\&F University,

Yangling 712100, Shaanxi, China

2 Institute of Water Saving Agriculture in Arid Areas of China, Northwest A\&F University, Yangling 712100, Shaanxi,

China

3 College of Plant Sciences and Technology, Huazhong Agricultural University, Wuhan 430070, Hubei, China 
Table 3 Effects of paclobutrazol on plant height, ear height and center of gravity height of maize in two growing seasons (2015-2016)

\begin{tabular}{|c|c|c|c|c|c|c|c|c|c|c|}
\hline \multirow[t]{2}{*}{ Treatments } & \multicolumn{2}{|c|}{ Plant height $(\mathrm{cm})$} & \multicolumn{2}{|c|}{ Ear height $(\mathrm{cm})$} & \multicolumn{2}{|c|}{ Height above ear $(\mathrm{cm})$} & \multicolumn{2}{|c|}{ Ear ratio $(\%)$} & \multicolumn{2}{|c|}{$\begin{array}{l}\text { Centre of gravity } \\
(\mathrm{cm})\end{array}$} \\
\hline & 2015 & 2016 & 2015 & 2016 & 2015 & 2016 & 2015 & 2016 & 2015 & 2016 \\
\hline CK1 & $240.0 \mathrm{a}$ & $242.1 \mathrm{a}$ & $117.1 \mathrm{a}$ & $117.3 \mathrm{a}$ & $123.0 \mathrm{a}$ & $124.8 \mathrm{a}$ & $48.8 \mathrm{a}$ & $48.4 \mathrm{a}$ & $104.1 \mathrm{a}$ & $124.8 \mathrm{a}$ \\
\hline $\mathrm{S} 1$ & $217.4 \mathrm{~b}$ & $217.2 \mathrm{~b}$ & $101.2 \mathrm{~b}$ & $102.8 \mathrm{~b}$ & $116.2 \mathrm{~b}$ & $114.4 \mathrm{~b}$ & $46.5 \mathrm{~b}$ & $47.3 \mathrm{ab}$ & $89.9 \mathrm{~b}$ & $114.4 \mathrm{~b}$ \\
\hline $\mathrm{S} 2$ & $205.8 \mathrm{c}$ & $205.4 \mathrm{c}$ & $90.4 \mathrm{c}$ & $94.1 \mathrm{c}$ & $115.5 \mathrm{c}$ & $111.3 \mathrm{~b}$ & $43.9 \mathrm{c}$ & $45.8 \mathrm{bc}$ & $78.0 \mathrm{c}$ & $111.3 \mathrm{c}$ \\
\hline S3 & $192.7 \mathrm{~d}$ & $195.8 \mathrm{~d}$ & $84.0 \mathrm{c}$ & $85.4 \mathrm{~d}$ & $108.7 \mathrm{c}$ & $110.4 \mathrm{~b}$ & $43.5 \mathrm{c}$ & $43.7 \mathrm{c}$ & $70.7 \mathrm{~d}$ & $110.4 \mathrm{~d}$ \\
\hline $\mathrm{CK} 2$ & $227.3 \mathrm{a}$ & $242.4 \mathrm{a}$ & $116.9 \mathrm{a}$ & $120.8 \mathrm{a}$ & $110.4 \mathrm{a}$ & $121.6 \mathrm{a}$ & $51.4 \mathrm{a}$ & $49.8 \mathrm{a}$ & $110.4 \mathrm{a}$ & $121.6 \mathrm{a}$ \\
\hline D1 & $209.3 \mathrm{~b}$ & $215.3 \mathrm{~b}$ & $101.9 \mathrm{~b}$ & $103.0 \mathrm{~b}$ & $107.4 \mathrm{ab}$ & $112.2 \mathrm{~b}$ & $48.7 \mathrm{~b}$ & $47.9 \mathrm{ab}$ & $107.4 \mathrm{~b}$ & $112.2 \mathrm{~b}$ \\
\hline D2 & $196.8 \mathrm{c}$ & 201.2 c & $98.0 \mathrm{~b}$ & $95.2 \mathrm{c}$ & $98.8 \mathrm{bc}$ & $106.0 \mathrm{bc}$ & $49.8 \mathrm{ab}$ & $47.3 \mathrm{ab}$ & $98.8 \mathrm{c}$ & $106.0 \mathrm{c}$ \\
\hline D3 & $186.6 \mathrm{~d}$ & $190.7 \mathrm{~d}$ & $86.6 \mathrm{c}$ & $88.4 \mathrm{~d}$ & $100.0 \mathrm{c}$ & $102.3 \mathrm{c}$ & $46.4 \mathrm{c}$ & $46.3 \mathrm{~b}$ & $100.0 \mathrm{~d}$ & $102.3 \mathrm{~d}$ \\
\hline
\end{tabular}

The means were compared by using least significant difference test (LSD); means followed by the different letters within each column are significantly different at $(P<0.05)$. CK1, S1, S2, and S3 represent seed-soaking with paclobutrazol at the rate of $0,200,300$, and $400 \mathrm{mg} \mathrm{L}^{-1}$, respectively. CK2, D1, D2, and D3 represent seed-dressing with paclobutrazol at the rate of $0,1.5,2.5$, and $3.5 \mathrm{~g} \mathrm{~kg}^{-1}$ seed, respectively

Note: The ear ratio values were mistyped (previous values were standard deviation values), please consider the new values 